\title{
Vacancy-induced enhancement of thermal conductivity in graphene
}

\author{
S.E. Krasavin ${ }^{1, *}$ and V. A. Osipov ${ }^{1,+}$ \\ ${ }^{1}$ Bogoliubov Laboratory of Theoretical Physics, \\ Joint Institute for Nuclear Research, \\ 141980 Dubna, Moscow region, Russia
}

(Dated: September 25, 2018)

\begin{abstract}
It is shown that the experimentally observed increase of the Young's modulus in single-layer graphene with low density of point defects leads to a noticeable enhancement of the thermal conductivity in a wide temperature range.
\end{abstract}

PACS numbers:

*Electronic address: krasavin@theor.jinr.ru

$\dagger$ Electronic address: osipov@theor.jinr.ru 
Recently, the effect of mechanical stiffness augmentation in graphene by controlled creation of a low density of point vacancy defects through $\mathrm{Ar}^{+}$irradiation has been experimentally revealed [1]. It has been found that the Young's modulus $\left(\mathrm{E}_{2 D}\right)$ of the graphene membrane increases with increasing irradiation dose and reaches a maximum of $550 \mathrm{Nm}^{-1}$ at $0.2 \%$ defect content. For a higher defect content a decreasing $\mathrm{E}_{2 D}$ has been observed. This effect was attributed to suppression of the out-of-plane fluctuations by defects.

The atomic simulation shows that this effect is mainly originated from specific bonds distribution in the surrounded monovacancy defects [2]. Moreover, it has been shown that such unusual mechanical response is the feature of presence of specifically monovacancies, whereas other types of point defects such as divacancy, 555-777 and Stone-Wales defects lead to the ordinary degradation of the graphene mechanical stiffness [2].

Notice that this unusual behavior will affect other important properties of defected graphene. In this letter, we consider a possible impact of monovacancy defects at a tiny concentration on the phonon thermal conductivity of graphene. Physically, a growing number of point defects will enhance the phonon scattering thus leading to the reduction in the thermal conductivity. In our case, however, the increase of the Young's modulus will result in the increased sound velocities, which reduces anharmonic phonon-phonon scattering processes and, thereafter, enhances the thermal conductivity. These opposite effects will compete in a wide temperature range. The aim of our paper is to analyze the influence of point defects on the thermal conductivity of graphene within a phenomenological single-mode relaxation time approach with all important scattering mechanisms taken into account.

Let us start with the well-known definition of sound velocities for longitudinal $(L A)$ and transverse $(T A)$ phonon branches in isotropic case [3]

$$
v_{L A}=\sqrt{E_{2 D} / \rho\left(1-\sigma^{2}\right)}, \quad v_{T A}=\sqrt{E_{2 D} / 2 \rho(1+\sigma)},
$$

where $\sigma$ is the Poisson's constant, $\rho$ is the material density and the Young's modulus is assumed to be a function of the defect density $n_{\text {def }}$. The inset in Fig. 1 shows the fit to the measured $E_{2 D}$ as a function of defect concentration given in [1].

We use the Callaway's theory where three phonon normal processes are taken into consideration explicitly [4]. Notice that the important role of normal phonon scattering processes in graphite-like materials and graphene has been noted in [5-7]. Thus, we consider four main 
scattering mechanisms relevant for suspended graphene: sample border (rough boundary), point defects, three-phonon normal and umklapp processes. Within the relaxation-time approximation the total mean free path can be written as

$$
l_{t o t, \lambda}^{-1}(q)=l_{0}^{-1}+l_{p d, \lambda}^{-1}(q)+l_{N, \lambda}^{-1}(q)+l_{U, \lambda}^{-1}(q)
$$

where $l_{0}, l_{p d, \lambda}, l_{N, \lambda}$ and $l_{U, \lambda}$ come from sample border, point defects, three phonon normal and umklapp scattering, respectively, for a given phonon branch $\lambda=(L A, T A, Z A)$ with the wave vector $q$. The mean free path due to normal processes is written as (see, e.g., [5], 7])

$$
l_{N, \lambda}^{-1}(q)=B_{N, \lambda} \omega_{\lambda}^{2}(q) T^{3}
$$

and for umklapp phonon scattering processes we employ a parametrized expression in the form

$$
l_{U, \lambda}^{-1}(q)=B_{U, \lambda} T^{3} \omega_{\lambda}^{2}(q) \exp \left(-\Theta_{\lambda} / 3 T\right)
$$

where $B_{N, \lambda}$ and $B_{U, \lambda}$ are parameters and $\Theta_{\lambda}$ is the Debye temperature. It should be mentioned that in our case $B_{(N, U) \lambda}=\bar{B}_{(N, U) \lambda} / v_{\lambda}$, and the numerical values of the parameters $\bar{B}_{N, \lambda}$ and $\bar{B}_{U, \lambda}$ are taken from [5]: $\bar{B}_{N, \lambda}=2.12 \times 10^{-25} s \mathrm{~K}^{-3}, \bar{B}_{U, \lambda}=3.18 \times 10^{-25} s \mathrm{~K}^{-3}$ for $\lambda=(L A, T A)$ and $\bar{B}_{N, \lambda}=1.48 \times 10^{-22} s \mathrm{~K}^{-3}, \bar{B}_{U, \lambda}=2.23 \times 10^{-22} s \mathrm{~K}^{-3}$ for $\lambda=(Z A)$.

The boundary scattering is expressed as

$$
l_{0}^{-1}=\frac{1}{d}
$$

with $d$ being the effective length determined from the geometry of the graphene sample [5]. The mean free path due to phonon-point defect scattering is taken to be

$$
l_{p d, \lambda}^{-1}(q)=\frac{S_{0} \Gamma}{4} \frac{q \omega_{\lambda}^{2}(q)}{v_{\lambda}^{2}}
$$

where $S_{0}$ is the cross-section area per one atom of the lattice, $\Gamma \approx\left(n_{\text {def }} / 2\right) \times 10^{-15} \mathrm{~cm}^{-2}$ is the mass-fluctuation phonon-scattering parameter (notice that $1 \%$ of vacancies corresponds to $\left.n_{\text {def }}=2 \times 10^{13} \mathrm{~cm}^{-2}[7]\right)$.

Within Callaway's formalism, the diagonal components of the thermal conductivity tensor $\kappa(T)$ can be presented by the sum of the Debye term

$$
\kappa_{D}(T)=\frac{\hbar^{2}}{S_{0} k_{B} T^{2}} \sum_{\lambda} \int d \omega \omega_{\lambda}^{2}(q) l_{t o t, \lambda}(\omega) v_{\lambda} C_{p h, \lambda}(\omega) N_{\lambda}(\omega)
$$


and the normal-drift term

$$
\kappa_{N}(T)=\frac{\hbar^{2}}{S_{0} k_{B} T^{2}} \sum_{\lambda} \frac{\left[\int d \omega \omega_{\lambda}^{2}(q) l_{t o t, \lambda}(\omega) l_{N, \lambda}^{-1}(\omega) v_{\lambda}^{2} C_{p h, \lambda}(\omega) N_{\lambda}(\omega)\right]^{2}}{\int d \omega \omega_{\lambda}^{2}(q)\left(1-l_{t o t, \lambda}(\omega) l_{N, \lambda}^{-1}(\omega)\right) l_{N, \lambda}^{-1}(\omega) v_{\lambda}^{3} C_{p h, \lambda}(\omega) N_{\lambda}(\omega)},
$$

where $C_{p h, \lambda}(\omega)=\exp \left(\hbar \omega_{\lambda}(q) / k_{B} T\right) /\left(\exp \left(\hbar \omega_{\lambda}(q) / k_{B} T\right)-1\right)^{2}, N_{\lambda}(\omega)$ is the density of states function per mole for each phonon branch, $k_{B}$ is the Boltzmann constant. Summation is performed over phonon polarization branches with the dispersion relations $\omega_{\lambda}(q)=q v_{\lambda}$ for $\lambda=L A, T A$. For out-of-plane (flexural) acoustic mode we use the dispersion law $\omega_{Z A}(q)=$ $q^{2} / 2 m$ [8] ( $m$ is an effective parameter taken here equal to $\left.320 \mathrm{sec} / \mathrm{cm}\right), l_{t o t, \lambda}(q, T)$ is the phonon mean free path given by Eqs.(2)-(6). The explicit form of $N_{\lambda}(\omega)$ is taken from [5].

Fig. 1 shows the calculated $\kappa(T)$ based on Eqs. (7) and (8) at the fixed concentration of vacancies $n_{\text {def }}=1.5 \times 10^{13} \mathrm{~cm}^{-2}$ for two cases: (a) the sound velocities do not depend on $n_{\text {def }}$ and have fixed values taken from [9]: $v_{L A}=21.3 \times 10^{5} \mathrm{~cm} / \mathrm{s}, v_{T A}=13.6 \times 10^{5}$ $\mathrm{cm} / \mathrm{s}$ (which corresponds to the Young's modulus $E_{2 D} \approx 360 \mathrm{Nm}^{-1}$ ), and (b) the sound velocities are calculated by Eq.(1). For chosen $n_{\text {def }}$ one has $E_{2 D} \approx 540 \mathrm{Nm}^{-1}$, so that

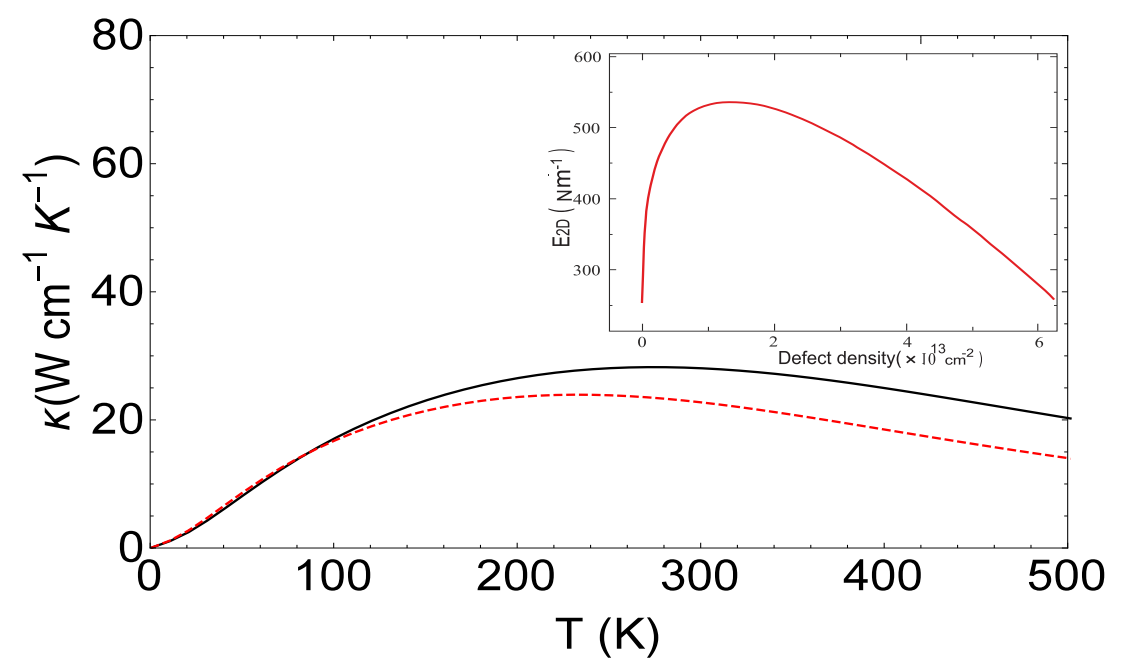

FIG. 1: Thermal conductivity versus temperature in a $2.9 \mu \mathrm{m}$ wide ribbon at $n_{\text {def }}=1.5 \times 10^{13}$ $\mathrm{cm}^{-2}$ in case of actual (solid line) and constant (dashed line) sound velocities. The insert shows a fit to the experimentally observed Young's modulus as a function of defect concentration (cf. Ref. [1]).

$v_{L A}=27.2 \times 10^{5} \mathrm{~cm} / \mathrm{s}$ and $v_{T A}=17.2 \times 10^{5} \mathrm{~cm} / \mathrm{s}$. As seen in Fig.1, in case of (b) markedly enhanced thermal conductivity takes place in a wide temperature range. The reason is quite 
clear because larger values of $v_{L A}$ and $v_{T A}$ lead to an increase of $l_{N, \lambda}(q)$ and $l_{U, \lambda}(q)$. At higher concentrations of vacancies, the difference between two cases (a) and (b) disappears which agrees with experimentally observed behavior of the Young's modulus (see the insert in Fig.1).

Fig. 2 shows the thermal conductivity as a function of $n_{\text {def }}$ at $T=300 \mathrm{~K}$. As is seen, the

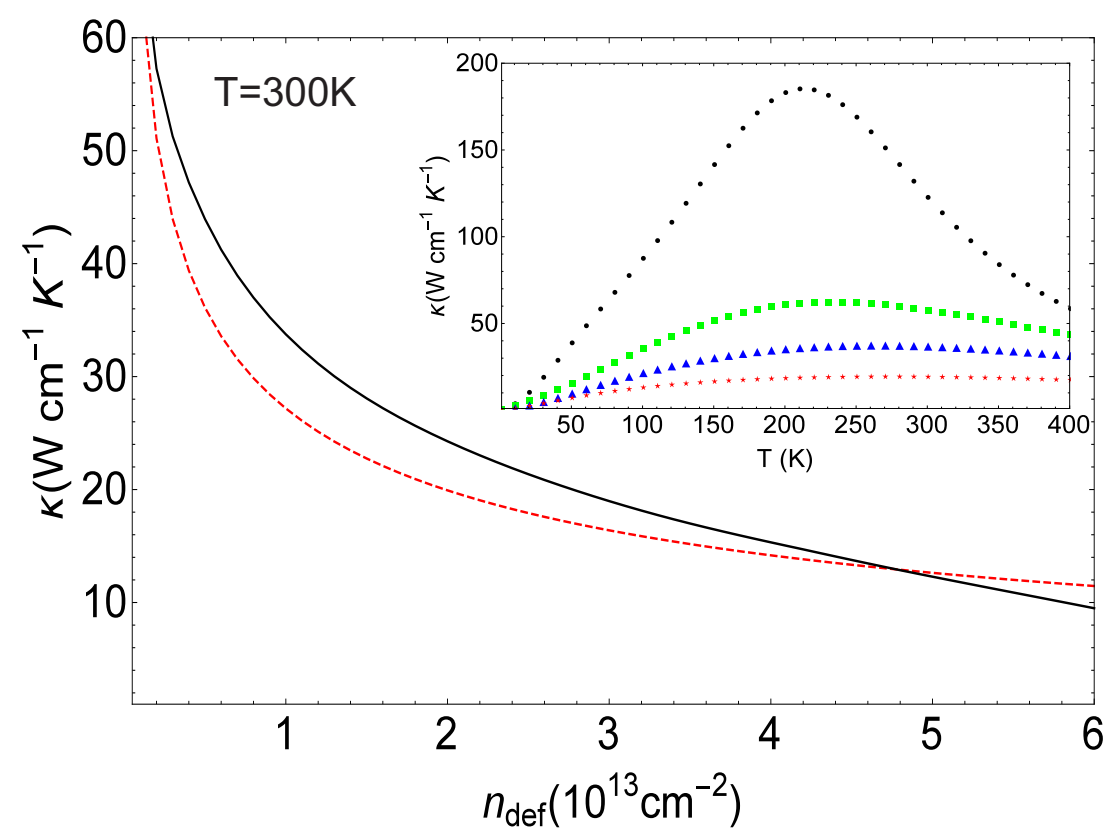

FIG. 2: Thermal conductivity versus the concentration of vacancies in a $2.9 \mu \mathrm{m}$ wide ribbon for actual (solid line) and constant (dashed line) sound velocities. The insert shows the calculated thermal conductivity versus temperature with the experimentally observed values of the Young's modulus for $n_{\text {def }}=0$ (circles), $2 \times 10^{12} \mathrm{~cm}^{-2}$ (squares), $8 \times 10^{12} \mathrm{~cm}^{-2}$ (triangles), $3 \times 10^{13} \mathrm{~cm}^{-2}$ (stars).

enhancement of $\kappa$ takes place in the range of $2.8 \times 10^{12} \mathrm{~cm}^{-2} \leq n_{\text {def }} \leq 5.3 \times 10^{13} \mathrm{~cm}^{-2}$ only. The explanation is as follows: at high temperatures the normal and umklapp scattering mechanisms are of the most importance and, in this case, $\kappa$ strongly depend on $v_{\lambda}$ (see, e.g., [9]). In turn, $v_{\lambda}$ is a function of $E_{2 D}$ in accordance with Eq. (1) and therefore depends on $n_{\text {def }}$ in this region. Accordingly, we have obtained the increased $\kappa$ at small concentrations of vacancies up to the value close to $5 \times 10^{13} \mathrm{~cm}^{-2}$ with the maximum gain at $n_{\text {def }} \sim 7 \times 10^{12}$ $\mathrm{cm}^{-2}$ which corresponds to the maximum of the Young's modulus.

Summarizing, we have found a marked increase in the thermal conductivity of graphene, 
which is a direct consequence of the experimentally observed effect of ultrahigh stiffness at low densities of vacancy defects. Our study shows that, in a limited range of defect concentrations, the thermal transport demonstrates a rather unique behavior. Namely, the growing number of defects provokes the enhancement of the thermal conductivity in a wide temperature range. Physically, this follows from the fact that after about $100 \mathrm{~K}$ the three-phonon scattering processes become dominant. They depend on the sound velocities which grow with the Young's modulus in some restricted region of $n_{\text {def }}$. This provides the enhancement of the thermal conductivity. Below $T \leq 100 \mathrm{~K}$ the main sources of the phonon scattering are sample border and point defects so that a strong increase in graphene stiffness has no effect on the thermal conductivity. Notice that our finding can be of importance in development of graphene-based thermoelectric devices.

[1] Lopez-Polin G, Gomez-Navarro C, Parente V, Guinea F, Katsnelson M I, Perez-Murano F, and Gomez-Herrero J. Increasing the elastic modulus of graphene by controlled defect creation. Nat Phys 2015;11: 2631.

[2] Kvashnin D G and Sorokin P B. Effect of Ultrahigh Stiffness of Defective Graphene from Atomistic Point of View. J Phys Chem Lett 2015;6: 238487.

[3] Munoz E, Lu J, and Yakobson B I. Ballistic Thermal Conductance of Graphene Ribbons. Nano Lett 2010;10(5): 165256.

[4] Callaway J. Model for Lattice Thermal conductivity at Low Temperatures. Phys Rev 1959;113(4): 104651.

[5] Alofi A and Srivastava G P. Thermal conductivity of graphene and graphite. Phys Rev B 2013;87: 1154219 .

[6] Slack G A and Galginaitis S. Thermal conductivity and Phonon Scattering by Marnetic impurities in CdTe. Phys Rev 1964;133: A253 268.

[7] Morelli D T, Heremans J P, and Slack G A. Estimation of the isotope effect on the lattice thermal conductivity of group IV and group III-V semiconductors. Phys Rev B 2002;66: 195304 9.

[8] Mounet N and Marzari N. First-principles determination of the structural, vibrational and thermodynamic properties of diamond, graphite, and derivatives. Phys Rev B 2005;71: 205214 
14.

[9] Nika D L, Pokatilov E P, Askerov A S, and Balandin A A. Phonon thermal conduction in graphene: Role of Umklapp and edge roughness scattering. Phys Rev B 2009;79: 15541312.

[10] Ng T Y, Yeo J J, and Liu Z S. A molecular dynamics study of the thermal conductivity of graphene nanoribbons containing dispersed Stone-Thrower-Wales defects, Carbon 2012;50: 488793. 